\title{
What is the best myocardial perfusion protocol in diabetic patients?
}

\author{
Leo H. B. Baur
}

Received: 30 June 2007 / Accepted: 4 July 2007 / Published online: 1 September 2007

(C) Springer Science+Business Media B.V. 2007

According to estimations of the World Health Organisation, the prevalence of diabetes mellitus in adults will rise from $4 \%$ in 1995 to 5,4\% in 2025 [1]. This will give a substantial impact on the cardiovascular health care of these patients. Several studies showed, that the presence of diabetes mellitus is independently associated with a doubling of cardiovascular risk in men and a threefold increase of cardiovascular risk in women and accounts for about $70 \%$ of all deaths in these patients [2,3]. Death in diabetic patients is mainly attributable to coronary artery disease, left ventricular hypertrophy and left ventricular dysfunction [1]. Coronary artery disease is the number one killer in diabetic patients. Diabetic patients are having coronary artery disease at a younger age, more diffuse and more extensively [46]. Besides this, diabetic patients have also an increased risk to develop congestive heart failure compared to a non-diabetic population [7]. Several pathophysiologic mechanisms are responsible for this: The higher incidence of ischemic heart disease in diabetes, the presence of hypertension and the development of specific diabetic cardiomyopathy [8].

L. H. B. Baur ( $\square)$

Department of Cardiology, Atrium Medical Centre Parkstad, Henri Dunantstraat 5, Heerlen 6401 CX, The Netherlands

e-mail: 1br01@atriummc.nl
Because silent ischemia is present in up to $60 \%$ of patients with diabetes, non-invasive imaging with stress echocardiography nuclear perfusion imaging or stress MRI should be used liberally in these patients before cardiovascular events occur [9]. One can argue, which technique should be used in selected patients. In this issue of the International Journal of Cardiac Imaging Ömür et al. showed that if nuclear imaging is used, in diabetic patients the results of imaging with Thallium 201 and TC-99m Sestamibi are comparable [10]. This in agreement with earlier studies performed by Cramer et al. [11] in 1994. He showed, that in 38 patients referred for evaluation of chest pain, and had to coronary angiography, the accuracy of Tc-99 m-sestamibi SPECT and TI-201 SPECT in detecting significant coronary artery disease was $87 \%$. In this study, only two patients were classified differently by the two methods. So, no clinically relevant differences in diagnostic accuracy were demonstrated between Tc-99 m-sestamibi and TI-201 SPECT using combined dipyridamole-exercise stress for the evaluation of coronary artery disease.

Because the sensitivity and specificity of stress echocardiography and stress MRI [12,13] are close to the values of radionuclide imaging, the choice of the specific test is primarily dependent to the experience of the local centre and the habitus of the patient. The most important issue, is that silent ischemia is recognized in diabetic patients and treatment is started early!. 


\section{References}

1. King H, Aubert RE, Herman WH (1998) Global burden of diabetes, 1995-2025. Diabetes Care 21:1414-1431

2. Kannel WB, McGee DL (1979) Diabetes and cardiovascular disease: the Framingham study. JAMA 241:20352038

3. Laakso M (1999) Hyperglycemia and cardiovascular disease in type 2 diabetes. Diabetes 48:937-942

4. Dawson A, Struthers AD (2003) Screening for treatable left ventricular abnormalities in diabetic patients. Expert opinion. Biol Ther 3:107-112

5. Goraya TY, Leibson CL, Palumbo PL, Weston SA, Killian JM, Pfeifer EA, Jacobsen SJ, Freye RL, Roger VL (2002) Coronary artherosclerosis in diabetes mellitus. A population bases autopsy study. J Am Coll Cardiol 40:946-953

6. Butler R, MacDonald TM, Struthers AD, Morris AD (1998) The clinical implications of diabetic heart disease. Eur Heart J 19:1617-1627

7. Kannel WB, Hjortland M, Castelli WP (1974) Role of diabetes in congestive heart failure: the Framingam Study. Am J Cardiol 34:29-34

8. Giles TD, Sander GE (2004) Diabetes mellitus and heart failure: basic mechanisms, clinical features, and therapeutic considerations. Clin Cardiol 22:553-568
9. Scognamiglio R, Negut C, Ramondo A, Tiengo A, Avogaro A (2006) Detection of coronary artery disease in asymptomatic patients with type 2 diabetes meWegscheider K, Fleck Ellitus. J Am Coll Cardiol 47:65-71

10. Őműr Ö, Özcan Z, Argon M, Acar E (2007) A comparative evaluation of Tl-201 and Tc-99 $\mathrm{m}$ sestamibi myocardial perfusion spect imaging in diabetic patients. Int $\mathrm{J}$ Cardiac Imaging. doi:10.1007/s10554-007-9244-6

11. Cramer MJ, Verzijlbergen JF, Van der Wall EE, Niemeyer MG, Zwinderman AH, Ascoop CA, Pauwels EJ (1994) Head-to-head comparison between technetium-99 m-sestamibi and thallium-201 tomographic imaging for the detection of coronary artery disease using combined dipyridamole-exercise stress. Coron Artery Dis 5:787-792

12. Marwick T, Willemart B, D'Hondt AM, Baudhuin T, Wijns W, Detry JM, Melin J (1993) Selection of the optimal non-exercise stress for the evaluation of ischemic regional myocardial dysfunction and malperfusion. Comparison of dobutamine and adenosine using echocardiography and 99 mTc-MIBI single photon emission computed tomography. Circulation 87:345-354

13. Nagel E, Klein C, Paetsch I, Hettwer S, Schnackenburg B (2003) Magnetic resonance perfusion measurement s for the noninvasive detection of coronary artery disease. Circulation 108:432-437 